\title{
A Novel Active Contour Model for MRI Brain Segmentation used in Radiotherapy Treatment Planning
}

\author{
Ahmad Mostaar ${ }^{1}$, Mohammad Houshyari ${ }^{2}$, Saeedeh Badieyan ${ }^{1}$
}

${ }^{1}$ Department of Biomedical Engineering and Medical Physics, School of Medicine, Shahid Beheshti University of Medical Sciences, Tehran, Iran

${ }^{2}$ Department of Radiation Oncology, Shohada-e Tajrish Hospital, School of Medicine, Shahid Beheshti University of Medical Sciences, Tehran, Iran

\section{Type of article: Original}

\begin{abstract}
Introduction: Brain image segmentation is one of the most important clinical tools used in radiology and radiotherapy. But accurate segmentation is a very difficult task because these images mostly contain noise, inhomogeneities, and sometimes aberrations. The purpose of this study was to introduce a novel, locally statistical active contour model (ACM) for magnetic resonance image segmentation in the presence of intense inhomogeneity with the ability to determine the position of contour and energy diagram.

Methods: A Gaussian distribution model with different means and variances was used for inhomogeneity, and a moving window was used to map the original image into another domain in which the intensity distributions of inhomogeneous objects were still Gaussian but were better separated. The means of the Gaussian distributions in the transformed domain can be adaptively estimated by multiplying a bias field by the original signal within the window. Then, a statistical energy function is defined for each local region. Also, to evaluate the performance of our method, experiments were conducted on MR images of the brain for segment tumors or normal tissue as visualization and energy functions.

Results: In the proposed method, we were able to determine the size and position of the initial contour and to count iterations to have a better segmentation. The energy function for 20 to 430 iterations was calculated. The energy function was reduced by about 5and 7\% after 70 and 430 iterations, respectively. These results showed that, with increasing iterations, the energy function decreased, but it decreased faster during the early iterations, after which it decreased slowly. Also, this method enables us to stop the segmentation based on the threshold that we define for the energy equation.

Conclusion: An active contour model based on the energy function is a useful tool for medical image segmentation. The proposed method combined the information about neighboring pixels that belonged to the same class, thereby making it strong to separate the desired objects from the background.
\end{abstract}

Keywords: image segmentation; active contour model; intensity inhomogeneity; energy function; brain MRI

\section{Introduction}

Segmentation is a fundamental operation performed on images that divides the image into homogeneous segments or regions to facilitate the analysis of the images. It is the process of grouping pixels together, with each group having at least one or more features in common. The features may be brightness, color, motion, or texture (1). Brain image segmentation is quite complicated and challenging, but doing it accurately is very important for detecting tumors, edema, and necrotic tissues. Magnetic resonance imaging (MRI) is a popular and non-invasive procedure that can be used safely for brain imaging with high contrast. It is used to visualize detailed internal structures, and it gives high-quality, informative images about the inside structure of brain, making it easier to diagnose many diseases (2-5).

\section{Corresponding author:}

Saeedeh Badieyan, Department of Biomedical Engineering and Medical Physics, Shahid Beheshti University of Medical Sciences, Tehran, Iran. E-mail: s.badieyan@gmail.com

Received: August 28, 2015, Accepted: February 20, 2015, Published: May 2016

iThenticate screening: November 05, 2015, English editing: March 22, 2015, Quality control: May 02, 2016

(C) 2016 The Authors. This is an open access article under the terms of the Creative Commons Attribution-NonCommercialNoDerivs License, which permits use and distribution in any medium, provided the original work is properly cited, the use is non-commercial and no modifications or adaptations are made. 
Many promising methods have been proposed for image segmentation, such as region merging-based methods (6-7), graph-based methods (8-11), and active contour model (ACM)-based methods (12, 13). A contour is a parameterized curve in an image domain, and the mathematical problem of segmentation involves minimizing an energy function the argument of which is an entire contour. The edge-based ACM methods are applicable to images with intensity inhomogeneity, but serious boundary leakage can occur for images with weak boundaries, and they can't drive the contour to stop at the object's boundary. While the region-based ACM method aims to drive the curve to evolve through some region-based descriptors, it is assumed that the image's intensity is homogeneous, and, thus, the method is not suitable for images with intensity inhomogeneity (14-18).

Intensity inhomogeneity often occurs in real-world images, and the main challenges in medical image segmentation are unknown and irregular noise, inhomogeneity, poor contrast, and weak boundaries, which can lead to serious misclassifications by intensity-based segmentation algorithms that assume a uniform intensity (15, 19). Intensity inhomogeneity is usually associated with a smooth and spatially varying field (bias field), multiplying the constant true signal of the same object in the measured image $(17,18)$. By searching the image's local redundant information, a mapping from the original image domain to another domain is defined such that the intensity probability model is more robust to noise, and the overlapping of intensity is suppressed to some extent. Then, a statistical energy functional for the distribution of each local region in the transformed domain is devised $(20,21)$. The test image to be processed is loaded into MATLAB.

\section{Material and Methods}

\subsection{Statistical active contours model}

In (22), Mumford and Shah formulated the following energy function:

$E^{M S}(u, C)=\int_{\Omega}(I-u)^{2} d x+\mu \int_{\Omega \backslash C}|\nabla u|^{2} d x+v|C|$

Given an image $\mathrm{I}$, find a contour $\mathrm{C}$ in $\Omega \subset \mathrm{R}^{2}$, where $|\mathrm{C}|$ is the length of contour. The minimization of the above function results in an optimal contour $\mathrm{C}$ that segments the images and a piecewise smooth image $u$ that approximates the original image $\mathrm{u}_{0}\left(\mathrm{u}_{0}: \Omega \rightarrow R\right)$. But due to the unknown contour $\mathrm{C}$ of the lower dimension, minimization of function (1) is difficult. Many methods have been proposed to simplify or modify the function (1) $(18,23)$.

\subsection{Statistical Model of Intensity Inhomogeneity}

Let $\Omega$ be the image domain, $\mathrm{b}(\mathrm{x}): \Omega \rightarrow \mathrm{R}$ be an unknown bias field, $\mathrm{I}(\mathrm{x}): \Omega \rightarrow \mathrm{R}$ be the given image, $\mathrm{J}(\mathrm{x}): \Omega \rightarrow \mathrm{R}$ be the true signal to be restored, and $\mathrm{n}(\mathrm{x}): \Omega \rightarrow \mathrm{R}$ be noise. We consider the following model of intensity inhomogeneity $(17)$.

$I(X)=b(X) J(X)+n(X)$

In the image domain $\Omega$, the domain of the $\mathrm{i}^{\text {th }}$ object is represented by $\Omega_{\mathrm{i}}$. The true signal $\mathrm{J}(\mathrm{x})$ often is assumed to be a piecewise constant within each object domain. The bias field $b$ is smooth in the image domain $\Omega$. The noise $n$ is assumed to be Gaussian-distributed with a zero mean and variance $\sigma_{\mathrm{n}}{ }^{2}(24)$. The distribution corresponding to the object domain $\Omega_{\mathrm{i}}$ with using multiple Gaussian probability distributions to model the image intensity is (25):

$p\left(I(y) \mid \theta_{i}\right)=\frac{1}{\sqrt{2 \pi \sigma_{i}}} \exp \left(-\frac{\left(I(y)-\mu_{i}(X)\right)^{2}}{2 \sigma_{i}^{2}}\right)$

where $\mu_{\mathrm{i}}(\mathrm{x})$ is the spatially varying mean, and $\sigma_{\mathrm{i}}$ is the standard deviation.

Since $\mathrm{b}(\mathrm{x})$ varies slowly, it can be assumed to be a constant in a small window (17). Thus, we can assume that $\mu_{\mathrm{i}}(\mathrm{x})$ $\approx \mathrm{b}(\mathrm{x}) \mathrm{c}_{\mathrm{i}}$. We use $\theta_{\mathrm{i}}=\left\{\mathrm{c}_{\mathrm{i}}, \sigma_{\mathrm{i}}, \mathrm{b}\right\}$ and $\theta=\left\{\theta_{\mathrm{i}}, \mathrm{i}=1, \ldots, \mathrm{N}\right\}$ in our following discussion.

When $\mathrm{I}(\mathrm{x})$ falls into the tail of the Gaussian distribution, misclassification will occur. One approach to reduce the overlapping tail is to compress the profile of the distribution while keeping its mean fixed (15).

\subsection{Principle of the Proposed Method}

For each position $\mathrm{x}$ in the image domain, we denote by Ox a neighboring region center. Since there are $\mathrm{N}$ nonoverlapping objects in the image, with $\Omega_{\mathrm{i}}$ being the domain of the $\mathrm{i}^{\text {th }}$ object, the whole image domain $\Omega$ can be represented as $\Omega=\mathrm{U}_{\mathrm{i}-1}, \ldots, \mathrm{N} \Omega_{\mathrm{i}}$ with $\Omega_{\mathrm{i}} \cap \Omega_{\mathrm{j}}=\emptyset, \forall \mathrm{i} \neq \mathrm{j}$. A mapping T: I $\left(\mathrm{x} \mid \theta_{\mathrm{i}}\right) \rightarrow I\left(\mathrm{x} \mid \theta_{\mathrm{i}}\right)$ from the original image intensity domain $\mathrm{D}(\mathrm{T})$ to another domain $\mathrm{R}(\mathrm{T})$ is defined as follows:

$\mathcal{J}\left(X \mid \theta_{i}\right)=\frac{1}{m_{i}(X)} \sum_{y \in \Omega_{i} \cap \mathrm{O}_{x}} I\left(y \mid \theta_{i}\right)$ 
where $\mathrm{m}_{\mathrm{i}}(\mathrm{x})=\left\|\Omega_{\mathrm{i}} \cap \mathrm{O}_{\mathrm{x}}\right\|$. The intensity of pixel $\mathrm{x}$ is assumed to be distributed independently (15). Thus, $\forall I\left(\mathrm{x} \mid \theta_{\mathrm{i}}\right) \in \mathrm{R}(\mathrm{T})$, whereby the corresponding probability density function (PDF) is still a Gaussian distribution (26).

Since the intensity inhomogeneity demonstrates itself as a smooth intensity variation across an image (17), we can assume that $I\left(\mathrm{y} \mid \theta_{\mathrm{i}}\right) \approx I\left(\mathrm{x} \mid \theta_{\mathrm{i}}\right), \forall \mathrm{y} \in \Omega_{\mathrm{i}} \cap \mathrm{O}_{\mathrm{x}}$. Because the product of Gaussian PDFs is still Gaussian (26), we have:

$\prod_{y \in \Omega_{i} \cap O_{x}} p\left(I\left(y \mid \theta_{i}\right)\right)=p\left(I\left(x \mid \theta_{i}\right)\right)^{m_{i}(x)} \propto N\left(\mu_{i}, \frac{\sigma_{i}^{2}}{m_{i}(x)}\right)$

Therefore:

$p\left(\mathcal{J}\left(x \mid \theta_{i}\right)\right) \approx \prod_{y \in \Omega_{i} \cap \mathrm{O}_{x}} p\left(I\left(y \mid \theta_{i}\right)\right)$

If we let $D=\left\{\left(\mathcal{J}\left(x \mid \theta_{i}\right)\right), x \in \Omega, i=1, \ldots, N\right\}$, we have the following likelihood function for the $\mathrm{i}^{\text {th }}$ object (26):

$p\left(D \mid \theta_{i}\right)=\prod_{x \in \Omega} p\left(\mathcal{J}\left(x \mid \theta_{i}\right)\right)$

We construct the following joint likelihood function (21):

$p(D \mid \theta)=\prod_{i=1}^{N} p\left(D \mid \theta_{i}\right)=\prod_{i=1}^{N} \prod_{x \in \Omega} p\left(\mathcal{J}\left(x \mid \theta_{i}\right)\right)=\prod_{x \in \Omega} q(\mathcal{J}(x \mid \theta))$

where $\theta=\left\{\theta_{i}, i=1, \ldots, N\right\}$ and

$q\left(\mathcal{J}\left(x \mid \theta_{i}\right)\right)=\prod_{i=1}^{N} p\left(\mathcal{J}\left(x \mid \theta_{i}\right)\right)=\prod_{i=1}^{N} \prod_{y \in \Omega_{i} \cap \mathrm{O}_{x}} p\left(I\left(y \mid \theta_{i}\right)\right)$

Substituting Eq. (4) into Eq. (8), the equation can be re-written as a Gaussian distribution, as follows:

$q\left(\mathcal{J}\left(x \mid \theta_{i}\right)\right)=\prod_{i=1}^{N} p\left(\mathcal{J}\left(x \mid \theta_{i}\right)\right) \propto N(\mu, \gamma)$,

where:

$\mu=\gamma \sum_{i=1}^{N} \frac{m_{i}(x) \mu_{i}}{\sigma_{i}^{2}}$ and $\gamma^{-1}=\sum_{i=1}^{N} \frac{m_{i}(x)}{\sigma_{i}^{2}}$

Obviously, the joint likelihood function in Eq. (8) of each pixel (voxel) is composed of multiple classes of intensities; thus, by using Eq. (10), our model can yield a soft classification. Moreover, as can be seen from Eq. (4), the intensity in the transformed domain exploits the information about neighboring pixels that belong to the same class, so its classification result is less sensitive to noise and can result in a smoother border of the object. The energy functional $l(\theta)$ as the log-likelihood function with respect to $\mathrm{p}(\mathrm{D} \mid \theta)$ in Eq.(8) is defined as:

$l(\theta) \triangleq-\log p(D \mid \theta)=$ constant $-\sum_{i=1}^{N} \int_{\Omega} \int_{\Omega_{i} \cap o_{x}} \log \left(p\left(I\left(y \mid \theta_{i}\right)\right)\right) d x d y$

Let $\mathrm{K} \rho(\mathrm{x}, \mathrm{y})$ be the indicator function of region $\mathrm{O}_{\mathrm{x}}$ :

$\kappa_{\rho}(x, y)=\left\{\begin{array}{c}1,\|y-x\| \leq \rho \\ 0, \text { else }\end{array}\right.$

Using Eqs. (1) and (13) and eliminating the trivial constant term, $1(\theta)$ can be re-written as:

$l(\theta)=\sum_{i=1}^{N} \int_{\Omega} \int_{\Omega_{i}} \kappa_{\rho}(x, y)\left(\log \left(\sigma_{i}\right)+\frac{\left(I(y)-b(x) c_{i}\right)^{2}}{2 \sigma_{i}^{2}}\right) d y d x$

\subsection{Energy Functional Formulation using the Level Set Method}

One level set function $\varphi$ can represent only two regions, i.e., inside and outside the contour $\mathrm{C}$, as $\Omega+=\operatorname{inside}(\mathrm{C})=$ $\{\varphi>0\}$ and $\Omega-=\operatorname{outside}(\mathrm{C})=\{\varphi<0\}$, respectively. This is called the Two-Phase model.

Twophase: $\left\{\begin{array}{c}M_{1}(\Phi)=H(\phi) \\ M_{2}(\Phi)=1-H(\phi)\end{array}\right.$

$\Phi$ represents the set of the level set functions. Then, $l(\theta)$ can be re-written as:

$l(\theta, \Phi)=\sum_{i=1}^{N} \int_{\Omega} d_{i}(y) M_{i}(\Phi(y)) d y$,

where: $\quad d_{i}(y) \triangleq \int_{\Omega} \kappa_{\rho}(x, y)\left(\log \left(\sigma_{i}\right)+\frac{\left(I(y)-b(x) c_{i}\right)^{2}}{2 \sigma_{i}^{2}}\right) d x, N=2$. 


\subsection{Energy minimization and level set evolution formulations}

For minimization of energy functional (16) with respect to each variable in $\theta=\left\{c_{i}, b, \sigma_{i}, i=1, \ldots, N, N=2\right\}$ other variables should be fixed. Minimization with respect to $\mathrm{c}_{\mathrm{i}}$, denoted by $\tilde{c}_{i}$, is done as follows:

$\tilde{c}_{i}=\frac{\int\left(\kappa_{\rho} * b\right) I M_{i}(\Phi(y)) d y}{\int\left(\kappa_{\rho} * b^{2}\right) M_{i}(\Phi(y)) d y}$,

where $*$ denotes the convolution operator.

Minimization with respect to b, denoted by $\tilde{b}$, is as follows:

$$
\tilde{b}(X)=\frac{\sum_{i=1}^{N} \kappa_{\rho} *\left(I M_{i}(\Phi(X))\right) \cdot \frac{c_{i}}{\sigma_{i}^{2}}}{\sum_{i=1}^{N} \kappa_{\rho} *\left(M_{i}(\Phi(X))\right) \cdot \frac{c_{i}^{2}}{\sigma_{i}^{2}}}
$$

Note that $\tilde{b}$ is actually the normalized convolution (27), which naturally leads to a smooth approximation of the bias field $b$. Minimization with respect to $\sigma_{i}$, denotedby $\widetilde{\sigma}_{i}$, can be done as follows:

$\tilde{\sigma}_{i}=\sqrt{\frac{\iint \kappa_{\rho}(y, x) M_{i}(\Phi(y))\left(I(y)-b(x) c_{i}\right)^{2}}{\iint \kappa_{\rho}(y, x) M_{i}(\Phi(y)) d y d x}}$

Minimizing the energy functional $l(\tilde{\theta}, \Phi)$ with respect to $\varphi$, we have the corresponding gradient descent formulation as follows:

$\frac{\partial \phi}{\partial t}=-\frac{\partial E(\tilde{\theta}, \Phi)}{\partial \phi}=\left(\tilde{d}_{2}-\tilde{d}_{1}\right) \delta(\phi)$,

where $\delta(\varphi)$ is the Dirac functional.

For simplicity, after each iteration of level set evolution, the level set function using the following formulation is defined:

$\phi^{n+1}=\phi^{n}+\Delta t_{2} \cdot \nabla^{2} \phi^{n}$

$\Phi^{\mathrm{n}+1}=\mathrm{K} * \varphi^{\mathrm{n}}$, where $\mathrm{K}$ is either a Gaussian kernel (28) or a constant kernel (16), where $\varphi^{\mathrm{n}}$ represents the level set function yielded by Eq. (26) during the $\mathrm{n}^{\text {th }}$ iteration, $\nabla^{2}$ represents the Laplacian operator, and $\Delta \mathrm{t}^{2}$ represents the diffusion strength. The Laplacian operator $\nabla^{2} \varphi$ is approximated by $\nabla^{2} \varphi \approx \mathrm{K} * \varphi$, where $*$ is a convolution operator, and $\mathrm{K}$ is a kernel defined as:

$\mathrm{K}=\left[\begin{array}{ccc}0 & 1 & 0 \\ 1 & -4 & 1 \\ 0 & 1 & 0\end{array}\right]$

\section{Results}

In this section, we show that the proposed method can be applied to efficiently segment images. To evaluate the performance of our method, experiments were conducted on MR images with intensity inhomogeneity. Some results from these images are reported here. It is easy to choose the parameters in our model. We initialize $b=1, \sigma=i, i=$ $1, \ldots, \mathrm{N}$, and then the initialization of $\widetilde{c}_{i}, \mathrm{i}=1, \ldots, \mathrm{N}$, can be calculated by Eq. (17). We set the time step for level set evolution as $\Delta \mathrm{t}=1$, the time step for the regularization as $\Delta \mathrm{t}_{2}=0.1$, and $\varepsilon=1$.

Figure 1 shows that this method can be applied for four MR images with intensity inhomogeneity. At first, the contour is applied on the center of images, and the image is shown after some iterations. In the other images, we changed the position and size of the contour, and the segmentation results are shown. The ability to choose the position and size of the initial contour helps us to decrease the number of iterations and the calculations; therefore the program can be performed very much faster, and it determines the position of tumor with higher accuracy. In Figure 2, we compared the result of segmentations for two different sizes and positions of contour for the same picture and same iterations. The proposed model performs well and handles high non-uniformity without degrading the final segmentation results.

As mentioned above, this program tries to minimize the amount of energy required. We define the threshold value equal to 0.0001 for energy equation. If the iterations reach 1000 or the ratio of energy for the last five iterations to 
the five iterations before it becomes less than 0.0001, the program stops. However, this ratio can be changed by the operator. Figure 3 shows the diagram of energy for different iterations and different images. The DICOM images that contain inhomogeneity can be used.

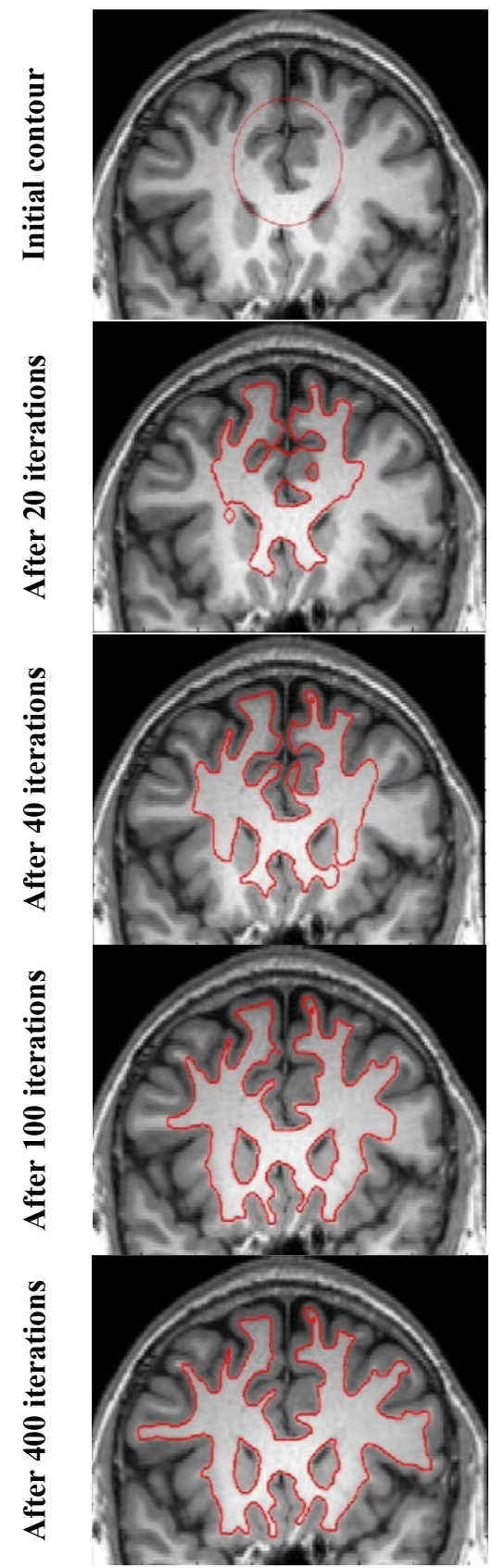

(a)
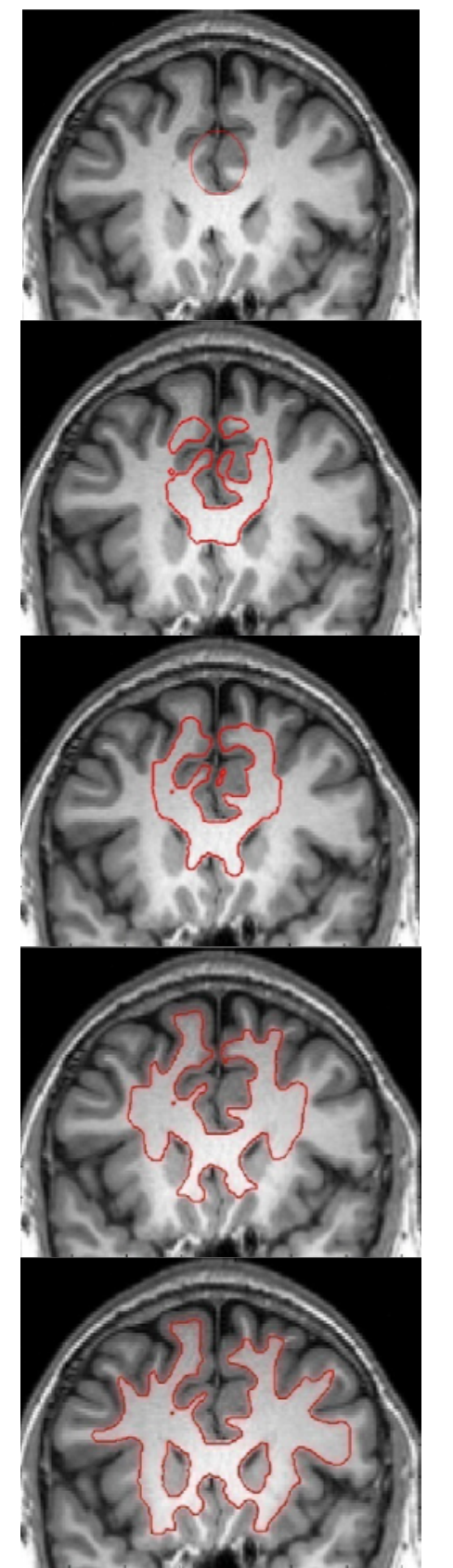

(b)
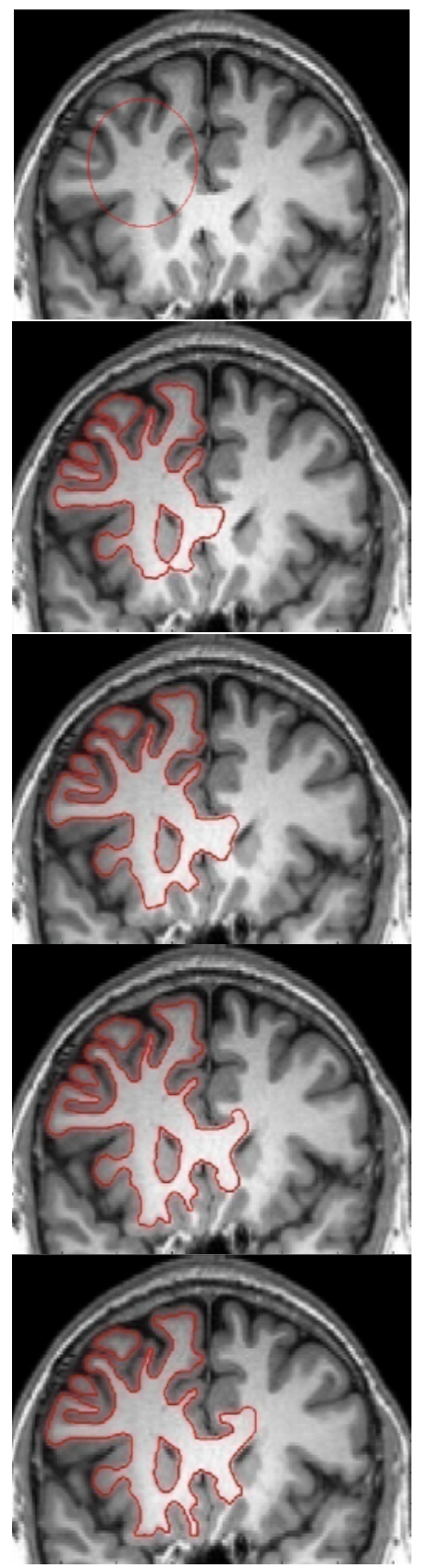

(c)

Figure 1. Segmentation with different size and different position of initial contour after 20,40,100, and 400 iterations: (a) Center (W/2, H/2), Radius = 20; (b) Center (W/2, H/2), Radius =10; (c) Center (W/3, H/2), Radius = 20 

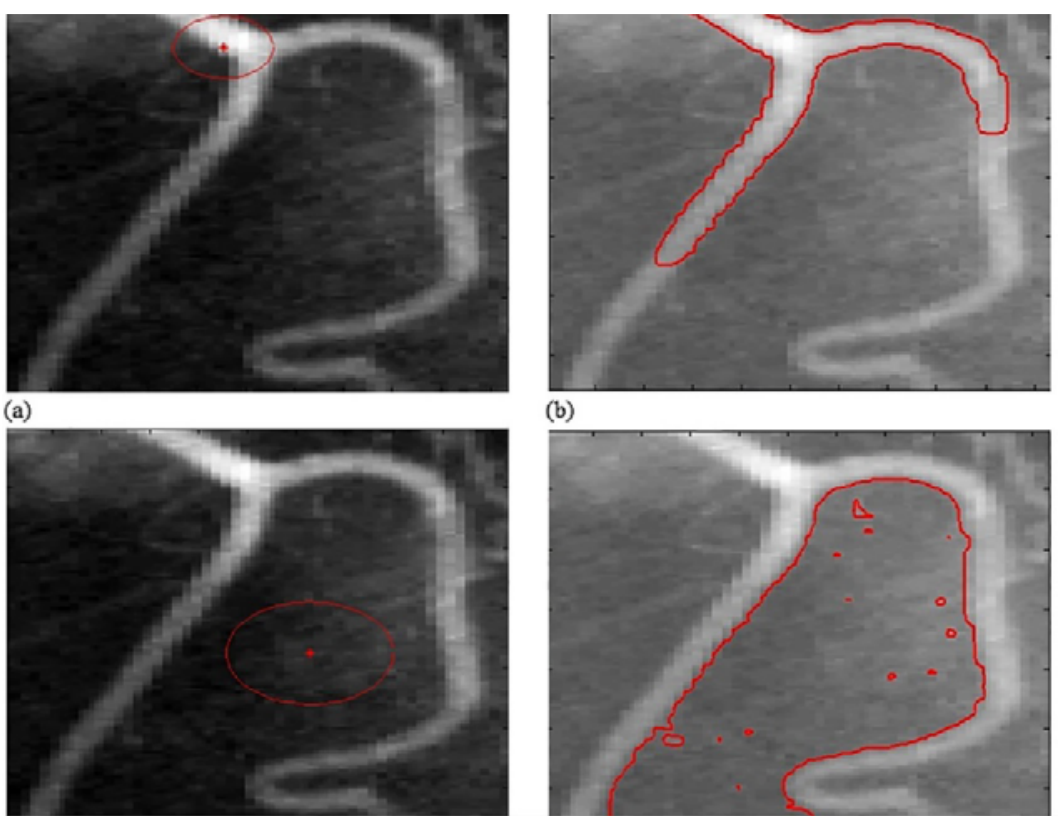

Figure 2. (a) Selecting a proper initial contour; (b) good segmentation; (c) an improper initial contour; (d) results fault segmentation

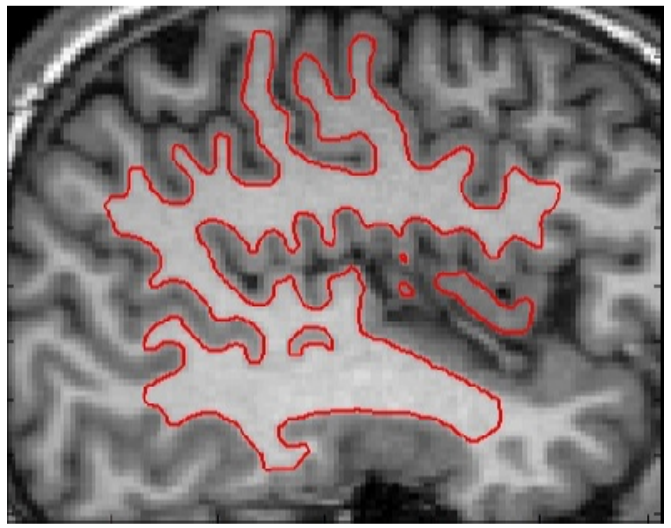

(a)

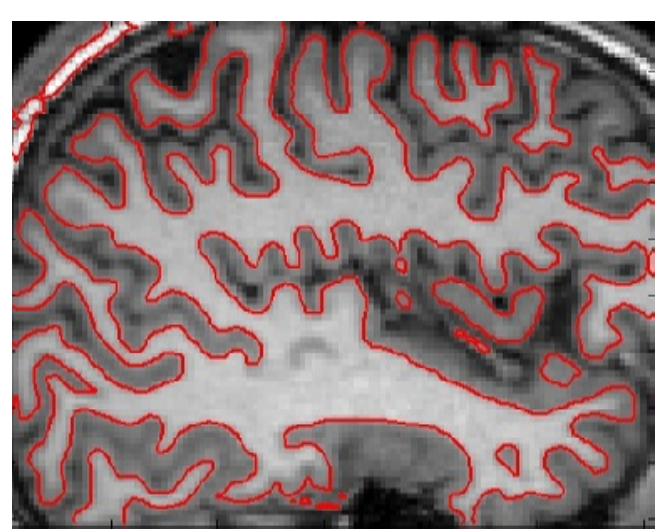

(c)

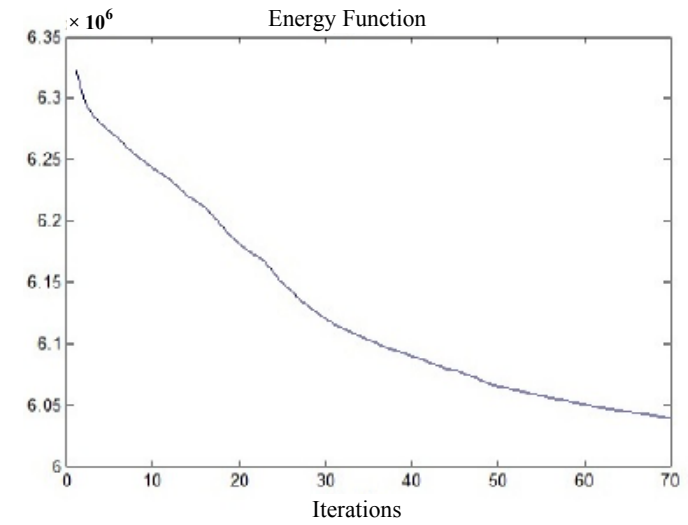

(b)

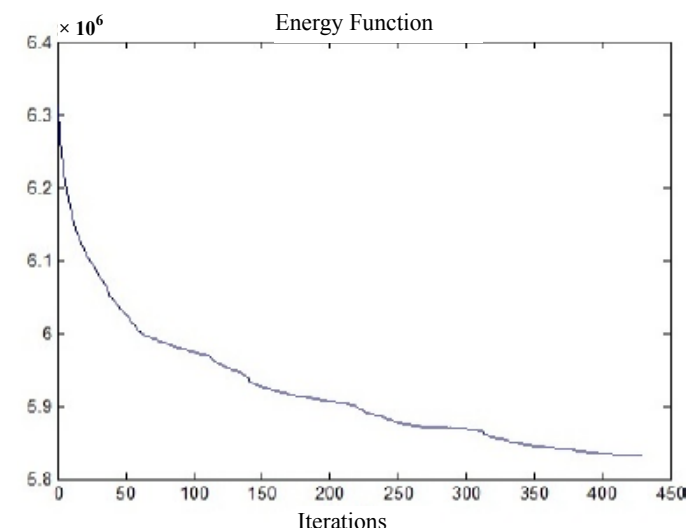

(d)

Figure 3. Segmentation and energy function versus iterations: (a, b) 70 iterations; (c, d) 430 iterations for the same images 


\begin{abstract}
4. Discussion
A large $\rho$ (the radius of initial contour) will increase the computational load in each iteration, while the convergence rate can be increased because information from larger regions is used. Besides that, we can choose the position of the contour and the threshold for energy equation. This helps us to segment tumor and other section of MRI with high accuracy, and it takes less time and fewer iterations than choosing the contour at the center of images. One of the well-known models for modeling images with intensity inhomogeneity that assumes an image with piecewise smooth is the Mumford-Shah (MS) model (22). The set of contours C that this model uses for separating different regions for low dimension is unknown and the problem is non-convex (23), therefore minimizing the energy functional of the MS model is difficult. But this limitation has been removed in our model. There are other verified models of the MS model, such as the seminal Chan-Vese (CV) model (18) and the piecewise-smoothing (PS) model $(23,30)$. All of these methods represent the contour C; however, the CV model is not applicable to images with intensity inhomogeneity because it models images by means of the piecewise constant. The PS model can yield a desirable segmentation for images with intensity inhomogeneity. However, the iteration of two partial differential equations (PDE) is needed in the PS model to approximate the original image. However, these iterations are very time consuming, so the practical application of the PS model is limited. Recently, some local region-based ACMs have been presented for images with inhomogeneous intensity, such as the local region descriptors (LRD) method (31), the local region-based (LRB) method (14), the local binary fitting (LBF) model $(32,33)$, the local intensity clustering (LIC) method (17), and the local region model (LRM) (34). However, there are some drawbacks with these local region-based models. The LRD model requires adjusting balloon force parameters for images with strongly overlapping intensity distribution, but the degree of the overlap is not defined exactly (31). Therefore, it is difficult to select a proper balloon force. The LRB method has two drawbacks. First, the Dirac functional used there is restricted to a neighborhood around the zero level set, which makes the level set evolution act locally. Therefore, the evolution can be easily trapped in local minima (18).
\end{abstract}

Second, the region descriptor in LRB is only based on the region mean information without considering the region variance, and this may lead to inaccurate segmentation. The second drawback of LRB also holds for the LBF model, because they use a similar energy functional. The LIC method can be considered as a locally weighted K-means clustering method (17). It does not consider the clustering variance, which may cause inaccurate segmentation; similar drawback exists for the K-means clustering based method in other research (35). The LRM method relates the local region statistics, i.e., local region means and variances, in interpreting the MS model. However, the local region means and variances are defined only empirically, rather than being derived from minimizing the MS energy. Comparing this method with the state-of-the-art LIC model (17) that is applicable for simultaneous segmentation and bias correction, it is apparent that, with an increase in noise level, the segmentation results by the LIC method will become very noisy; thus, the segmentation result using our method is much better. As shown in Figure 3, the energy function was reduced about 5 and $7 \%$ after 70 and 430 iterations, respectively. These results show that with increasing of iteration, the energy function decreased but in beginning iteration it decreased faster and then at large iteration the energy function decreased slowly and more iterations did not change the energy function significantly. Thus, it is possible to define a threshold for the energy function to stop the iterations. If differences between some sequence iterations are less than defined threshed, the energy function is almost constant. This property of the model makes it useful for automated segmentation that can be used in radiotherapy treatment planning systems that tumor and organ at risk organ must be segmented.

As discussed above, our model has the ability to segment the images that have high inhomogeneity and to do so faster and more accurately than other models that have been proposed. In other models, the accuracy decreases as inhomogeneity increases, but this decrease is negligible in our model. In addition, we can easily change the parameters based on images that help us to segment given images more accurately and more easily, especially for medical images of the brain, while this is a limitation for other methods.

\title{
5. Conclusions
}

In this paper, we presented a locally statistical active contour model for segmenting images with intensity inhomogeneity that provides the ability to determine the size and position of initial contour that makes the segmentation faster and more accurate. Our method combines the information about neighboring pixels that belong to the same class, which makes it capable of separating the desired objects from the background. Level set function makes this model useful for MR images segmentation. In our study, the determination of position and size of the 
contour and the threshold of the energy function are based on visual assessment. Finding a method for determining these objects is a suggestion for future work.

\section{Acknowledgments:}

This study was done in the Biomedical and Medical Physics Department at Shahid Beheshti University of Medical Sciences. The authors gratefully acknowledge the help and support of the Department's staff.

\section{Conflict of Interest:}

There is no conflict of interest to be declared.

\section{Authors' contributions:}

All authors contributed to this project and article equally. All authors read and approved the final manuscript.

\section{References:}

1) Bovik A. Handbook of image and video processing. Second edition. Elsevier Academic Press, 2005.

2) Tian D, Fan L. A brain MR images segmentation method based on SOM neural network. Bioinformatics and Biomedical Engineering. 2007; 686-9.doi:10.1109/ICBBE.2007.179.

3) Llado X, Oliver A, Cabezas M, Freixenet J, Vilanova J.C, Quiles A, et al. Segmentation of multiple sclerosis lesions in brain MRI: a review of automated approaches. Information Sciences. 2012; 186(1): 164-185.doi: 10.1016/j.ins.2011.10.011.

4) Devi, C.N, Chandrasekharan A, Sundararaman V.K, Alex Z.C. Neonatal brain MRI segmentation: A review. Journal of computers in biology and medicine. 2015; 64(1): 163-78. doi: 10.1016/j.compbiomed.2015.06.016, PMID: 26189155

5) Ji Z, Liu J, Cao G, Sun Q, Chen Q. Robust spatially constrained fuzzy c-means algorithm for brain MR image segmentation. Pattern Recognition. 2014; 47(7): 2454-66. doi: 10.1016/j.patcog.2014.01.017.

6) Peng, B, Zhang L, Zhang D. Automatic image segmentation by dynamic region merging. IEEE Trans. Image Process 2011; 20(12): 3592-605. doi: 10.1109/TIP.2011.2157512.

7) Talu, M.F. ORACM: Online region-based active contour model. Expert Systems with Applications. 2013; 40(16): 6233-40. doi: 10.1016/j.eswa.2013.05.056.

8) Minati L, Grisoli M, Seth A.K, Critchley H.D. Decision-making under risk: a graph-based network analysis using functional MRI. Neuroimage. 2012; 60(4): 2191-205. doi: 10.1016/j.neuroimage.2012.02.048, PMID: 22387471

9) Lai Z, Qu X, Liu Y, Guo D, Ye J, Zhan Z, et al. Image Reconstruction of Compressed Sensing MRI Using Graph-based Redundant Wavelet Transform. Medical Image Analysis. 2015; 1-27. doi: 10.1016/j.media.2015.05.012.

10) Peng B, Zhang L, Zhang D, Yang J. Image segmentation by iterated region merging with localized graph cuts. Pattern Recognition. 2011; 44(10): 2527-38. doi: 10.1016/j.patcog.2011.03.024

11) Egger J, Colen RR, Freisleben B, Nimsky C. Manual refinement system for graph-based segmentation results in the medical domain. Journal of Medical Systems. 2012; 36(5): 2829-39. doi: 10.1007/s10916011-9761-7, PMCID: PMC3691109

12) Kass M, Witkin A, Terzopoulos D. Snakes: active contour models. International Journal of Computer Vision. 1987; 1(4): 321-31. doi: 10.1007/BF00133570.

13) Moreno JC, Prasath VBS, Proença H, Palaniappan K. Fast and globally convex multiphase active contours for brain MRI segmentation. Computer Vision and Image Understanding. 2014; 125: 237-50. doi: 10.1016/j.cviu.2014.04.010.

14) Lankton S, Tannenbaum A. Localizing region-based active contours.. IEEE Trans. Image Process 2008; 17(11): 2029-39. doi: 10.1109/TIP.2008.2004611.

15) Zhu S, Yuille A. Region competition: unifying snakes, region growing, and Bayes/MDL for multiband image segmentation.. IEEE Trans. Pattern Anal. Mach. Intell. 1996; 18(9): 884-900. doi: $10.1109 / 34.537343$

16) Zhang K, Zhang L, Zhang S. A variational multiphase level set approach to simultaneous segmentation and bias correction. IEEE International Conference on Image Processing. 2010; 4105-08. doi: 10.1109/ICIP.2010.5651554.

17) Li C, Huang R, Ding Z, Gatenby C, Metaxas D, Gore JC. A level set method for image segmentation in the presence of intensity inhomogeneities with application to MRI.. IEEE Trans. Image Process. 2011; 20(7): 2007-16. doi: 10.1109/TIP.2011.2146190. 
18) Chan T, Vese L. Active contours without edges.. IEEE Trans. Image Process. 2001; 10(2): 266-77. doi: $10.1109 / 83.902291$.

19) Paul T.U, Bandhyopadhyay SK. Segmentation of Brain Tumor from Brain MRI Images Reintroducing KMeans with advanced Dual Localization Method. International Journal of Engineering Research and Applications. 2012; 2(3): 226-31.

20) Zhang K, Zhang L, Lam KM, Zhang D. A locally statistical active contour model for image segmentation with intensity inhomogeneity. Computer Vision and Pattern Recognition. 2013. arXiv preprint. arXiv: 1305.7053.

21) Gibou F, Fedkiw R, Eric FE. A fast hybrid k-means level set algorithm for segmentation. In 4th Annual Hawaii International Conference on Statistics and Mathematics. 2005; 281-291. Honolulu, Hawaii.

22) Mumford D, Shah J. Optimal approximation by piecewise smooth function and associated variational problems. Communications on Pure Applied Mathematics. 1989; 42(5): 577-685. doi: 10.1002/cpa.3160420503.

23) Vese L, Chan T. A multiphase level set framework for image segmentation using the mumford and shah model. International Journal of Computer Vision. 2002; 50(3): 271-293. doi: 10.1023/A:1020874308076.

24) Wang $Y$, Lei T. Statistical analysis of MR imaging and its applications in image modeling. IEEE International Conference of Image Processing and Neural Networks. 1994; 1: 866-70. doi: 10.1109/ICIP.1994.413438.

25) Brox T. From pixels to regions: partial differential equations in image analysis, Ph.D Thesis, Saarland University, Germany, 2005.

26) Duda R, Hart P., Stork D, Pattern classification. John Wiley \& Sons Inc press, 2nd edition, 2001.

27) Knutsson $\mathrm{H}$, Westin $\mathrm{C}$. Normalized and differential convolution: methods for interpolation and filtering of incomplete and uncertain data. IEEE Conference on Computer Vision and Pattern Recognition. 1993; 515 23. doi: 10.1109/CVPR.1993.341081.

28) Zhang K, Song H, Zhang L. Active contours driven by local image fitting energy. Pattern Recognition. 2010; 43(4): 1199-206. doi: 10.1016/j.patcog.2009.10.010.

29) Gonzalez R, Woods R, Digital image processing. Third edition, Prentice Hall, 2008.

30) Tsai A, Yezzi J A , Willsky AS, Curve evolution implementation of the Mumford-Shah functional for image segmentation, denoising, interpolation, and magnification.. IEEE Trans. Image Process. 2001; 10(8): 1169-1186. doi: 10.1109/83.935033.

31) Darolti C, Mertins A, Bodensteiner C, Hofmann UG. Local region descriptors for active contours evolution.. IEEE Trans. Image Process. 2008; 17(12): 2275-88. doi: 10.1109/TIP.2008.2006443.

32) Li C, Chiu-Yen K, Gore JC, Ding Z. Minimization of region-scalable fitting energy for image segmentation.. IEEE Trans. Image Process. 2008; 17(10): 1940-9. doi: 10.1109/TIP.2008.2002304.

33) Li C, Chiu-Yen K, Gore JC, Ding Z. Implicit active contours driven by local binary fitting energy. IEEE Conference on Computer Vision and Pattern Recognition. 2007; 1-7. doi: 10.1109/CVPR.2007.383014.

34) Brox T, Cremers D. On local region models and a statistical interpretation of the piecewise smooth Mumford-Shah functional. International journal of computer vision. 2009; 84(2): 184-93. doi: 10.1007/s11263-008-0153-5.

35) Bishop CM. Pattern Recognition and Machine Learning. Springer-Verlag, New York, 2006. 